\title{
On the generalized fractional derivatives and their Caputo modification
}

\author{
Fahd Jarad ${ }^{\mathrm{a}}$, Thabet Abdeljawad ${ }^{\mathrm{b}}$, Dumitru Baleanu $\mathrm{A}^{\mathrm{a}, \mathrm{c}, *}$ \\ a Mathematics Department, Faculty of Arts and Sciences, Çankaya University, 06790, Etimesgut, Ankara, Turkey. \\ ${ }^{b}$ Department of Mathematics and Physical Sciences, Prince Sultan University, P. O. Box 66833, 11586 Riyadh, Saudi Arabia. \\ ${ }^{c}$ Institute of Space Sciences, Magurele-Bucharest, Romania.
}

Communicated by X. J. Yang

\begin{abstract}
In this manuscript, we define the generalized fractional derivative on $A C_{\gamma}^{n}[a, b]$, the space of functions defined on [a, $\left.b\right]$ such that $\gamma^{n-1} f \in A C[a, b]$, where $\gamma=x^{1-\rho} \frac{d}{d x}$. We present some of the properties of generalized fractional derivatives of these functions and then we define their Caputo version. (C)2017 All rights reserved.
\end{abstract}

Keywords: Riemann-Liouville fractional derivatives, Caputo fractional derivatives, Hadamard fractional derivatives, Caputo-Hadamard fractional derivatives, generalized fractional integral, generalized Caputo fractional derivatives. 2010 MSC: 26A33, 34A08.

\section{Introduction and preliminaries}

The fractional calculus is an important developing field in both pure and applied mathematics [16, 20, 21]. Many real world problems have been investigated within the fractional derivatives, particularly Caputo fractional derivative is extensively and successfully used in many branches of sciences and engineering [11, 18-20]. We recall that the system having memory effect are better described within fractional differential operators mainly due to the non-locality of these operators [11, 16, 19-21]. However, the non-locality has various forms. Therefore, the researchers try to generalize the fractional operators to capture the hidden aspects of the real non-local phenomena. On the other hand, many researchers work on fractional integrals and derivatives with non-local and non-singular kernels [7, 9, 17, 22, 23]. One of the trends in fractional is the discrete fractional operators which are proved to have good applications in various fields $[1-4,6,10]$.

From the classical fractional calculus, we recall [16, 20, 21].

- The left Riemann-Liouville fractional integral of order $\alpha>0$ starting from a has the following form

$$
\left({ }_{a} I^{\alpha} f\right)(x)=\frac{1}{\Gamma(\alpha)} \int_{a}^{x}(x-t)^{\alpha-1} f(t) d t .
$$

\footnotetext{
${ }^{*}$ Corresponding author

Email addresses: fahd@cankaya.edu.tr (Fahd Jarad), tabdeljawad@psu.edu.sa (Thabet Abdeljawad), dumitru@cankaya.edu.tr (Dumitru Baleanu)
}

doi:10.22436/jnsa.010.05.27 
- The right Riemann-Liouville fractional integral of order $\alpha>0$ ending at $b>a$ is defined by

$$
\left(I_{b}^{\alpha} f\right)(x)=\frac{1}{\Gamma(\alpha)} \int_{x}^{b}(t-x)^{\alpha-1} f(t) d t .
$$

- The left Riemann-Liouville fractional derivative of order $\alpha>0$ starting at a is given below

$$
\left({ }_{a} D^{\alpha} f\right)(x)=\left(\frac{d}{d x}\right)^{n}\left({ }_{a} I^{n-\alpha} f\right)(x), \quad n=[\alpha]+1 .
$$

- The right Riemann-Liouville fractional derivative of order $\alpha>0$ ending at $b$ becomes

$$
\left(D_{b}^{\alpha} f\right)(x)=\left(-\frac{d}{d x}\right)^{n}\left(I_{b}^{n-\alpha} f\right)(x)
$$

- The left Caputo fractional of order $\alpha>0$ starting from a has the following form

$$
\left({ }_{a} D^{\alpha} f\right)(x)=\left({ }_{a} I^{n-\alpha} f^{(n)}\right)(x), \quad n=[\alpha]+1 .
$$

- The right Caputo fractional derivative of order $\alpha>0$ ending at $b$ becomes

$$
\left(D_{b}^{\alpha} f\right)(x)=\left(I_{b}^{n-\alpha}(-1)^{n} f^{(n)}\right)(x) .
$$

The Hadamard type fractional integrals and derivatives were introduced in [15] as:

- The left Hadamard fractional integral of order $\alpha>0$ starting from a has the following form

$$
\left(a g^{\alpha} f\right)(x)=\frac{1}{\Gamma(\alpha)} \int_{a}^{x}(\ln x-\ln t)^{\alpha-1} f(t) d t .
$$

- The right Hadamard fractional integral of order $\alpha>0$ ending at $b>a$ is defined by

$$
\left(\partial_{\mathrm{b}}^{\alpha} \mathrm{f}\right)(\mathrm{x})=\frac{1}{\Gamma(\alpha)} \int_{\mathrm{x}}^{\mathrm{b}}(\ln \mathrm{t}-\ln \mathrm{x})^{\alpha-1} \mathrm{f}(\mathrm{t}) \mathrm{dt} .
$$

- The left Hadamard fractional derivative of order $\alpha>0$ starting at $a$ is given below

$$
\left(a_{a} D^{\alpha} f\right)(x)=\left(x \frac{d}{d x}\right)^{n}\left(a I^{n-\alpha} f\right)(x), \quad n=[\alpha]+1 .
$$

- The right Hadamard fractional derivative of order $\alpha>0$ ending at $b$ becomes

$$
\left(\mathcal{D}_{\mathrm{b}}^{\alpha} f\right)(x)=\left(-x \frac{\mathrm{d}}{\mathrm{d} x}\right)^{n}\left(I_{b}^{n-\alpha} f\right)(x) .
$$

The authors in $[8,12]$ defined the Caputo-Hadamard fractional derivatives as:

- The left Caputo-Hadamard fractional of order $\alpha>0$ starting from a has the following form

$$
\left({ }_{a}^{C} \mathcal{D}^{\alpha} f\right)(x)={ }_{a} \mathcal{D}^{\alpha}\left[f(t)-\sum_{k=0}^{n-1} \frac{\delta^{k} f(a)}{k !}\left(\log \frac{t}{a}\right)^{k}\right](x), \quad \delta=x \frac{d}{d x},
$$

and in the space $A C_{\delta}^{n}[a, b]=\left\{g:[a, b] \rightarrow C: \delta^{n-1}[g(x)] \in A C[a, b]\right\}$ equivalently by

$$
\left({ }_{a}^{C} \mathcal{D}^{\alpha} f\right)(x)=\left(a_{a} g^{n-\alpha}\left(x \frac{d}{d x}\right)^{n} f\right)(x), \quad n=[\alpha]+1 .
$$


- The right fractional derivative of order $\alpha>0$ ending at $b$ was defined by

$$
\left({ }_{a}^{c} \mathcal{D}^{\alpha} f\right)(x)={ }_{a} \mathcal{D}^{\alpha}\left[f(t)-\sum_{k=0}^{n-1} \frac{(-1)^{k} \delta^{k} f(a)}{k !}\left(\log \frac{b}{t}\right)^{k}\right](x),
$$

and the space $A C_{\delta}^{n}[a, b]$ equivalently by

$$
\left(\mathcal{D}_{b}^{\alpha} f\right)(x)=\left(\mathcal{J}_{b}^{n-\alpha}\left(-x \frac{d}{d x}\right)^{n} f\right)(x) .
$$

For $a<b, c \in \mathbb{R}$ and $1 \leqslant p<\infty$, define the function space

$$
X_{c}^{p}(a, b)=\left\{f:[a, b] \rightarrow \mathbb{R}:\|f\|_{X_{c}^{p}}=\left(\int_{a}^{b}\left|t^{c} f(t)\right|^{p} \frac{d t}{t}\right)^{1 / p}<\infty\right\} .
$$

For $p=\infty,\|f\|_{X_{c}^{p}}=\operatorname{ess~sup}_{a \leqslant t \leqslant b}\left[t^{c}|f(t)|\right]$. The generalized left and right fractional integrals (in the sense of Katugampola) are defined by [13]

$$
\left({ }_{a} I^{\alpha, \rho} f\right)(x)=\frac{1}{\Gamma(\alpha)} \int_{a}^{x}\left(\frac{x^{\rho}-t^{\rho}}{\rho}\right)^{\alpha-1} f(t) \frac{d t}{t^{1-\rho}}
$$

and

$$
\left(I_{b}^{\alpha, \rho} f\right)(x)=\frac{1}{\Gamma(\alpha)} \int_{x}^{b}\left(\frac{t^{\rho}-x^{\rho}}{\rho}\right)^{\alpha-1} f(t) \frac{d t}{t^{1-\rho}}
$$

respectively. In [13] it was shown that the integral operator $I^{\alpha, \rho}$ is bounded on the function space $X_{c}^{p}(a, b)$, $\rho-1 \geqslant c$. Indeed,

$$
\left\|I_{a}^{\alpha, \rho} f\right\|_{X_{c}^{p}} \leqslant K\|f\|_{X_{c}^{p}}, \quad K=\frac{b^{\alpha \rho-1}}{\Gamma(\alpha)} \int_{1}^{b / a} u^{c-\alpha \rho-1}\left(\frac{u^{\rho}-1}{\rho}\right)^{\alpha-1} d u, \quad \rho \neq 0 .
$$

Also, the semigroup property

$$
{ }_{a} I^{\alpha, \rho}{ }_{a} I^{\mu, \rho} \rho_{f}{ }_{a} I^{\alpha+\mu, \rho} f, \quad f \in X_{c}^{p}(a, b), \quad \alpha>0, \quad \mu>0, \quad 1 \leqslant p<\infty, \quad a \in(0, \infty), \quad \rho, c \in \mathbb{R}, \quad \rho \geqslant c .
$$

The left and right generalized fractional derivatives of order $\alpha$ are defined by [14]

$$
\left({ }_{a} D^{\alpha, \rho} f\right)(x)=\gamma^{n}\left({ }_{a} I^{n-\alpha, \rho} f\right)(x)=\frac{\gamma^{n}}{\Gamma(n-\alpha)} \int_{a}^{x}\left(\frac{x^{\rho}-t^{\rho}}{\rho}\right)^{n-\alpha-1} f(t) \frac{d t}{t^{1-\rho}}
$$

and

$$
\left(D_{b}^{\alpha, \rho} f\right)(x)=(-\gamma)^{n}\left({ }_{a} I^{n-\alpha, \rho} f\right)(x)=\frac{(-\gamma)^{n}}{\Gamma(n-\alpha)} \int_{x}^{b}\left(\frac{t^{\rho}-x^{\rho}}{\rho}\right)^{n-\alpha-1} f(t) \frac{d t}{t^{1-\rho}},
$$

respectively, where $\rho>0$.

The authors in [5] did define the Caputo version of the generalized fractional derivatives. From the mathematical view, we have to consider the fractional derivatives of functions belonging to specific spaces. In this aspect, this will help us to treat efficiently the numerical solutions of differential equations involving the generalized fractional derivatives. The main purpose of this article is to present the generalized fractional derivatives of absolute differentiable continuous and differentiable continuous functions and consider some of their properties that will lead us to define the Caputo modification of these derivatives.

The organization of the paper is as follows. In Section 2, we present the generalized fractional derivatives of functions in the spaces $A C_{\gamma}^{n}[a, b]$ and $C_{\gamma}^{n}[a, b]$. In Section 3, we define the Caputo version of generalized fractional derivatives. Section 4 contains the conclusion. 


\section{The generalized fractional derivatives on the space $A C_{\gamma}^{\mathfrak{n}}[a, b]$ and $C_{\gamma}^{\mathfrak{n}}[a, b]$}

From basic analysis we recall that $f \in A C[a, b]$ is the space of absolutely continuous functions on $[a, b]$ if and only if $f(x)=c+\int_{a}^{x} \varphi(t) d t, \varphi(t) \in L(a, b)$ (see also [16, 1.1.5]). Let's denote the space of all continuous real (Banach-) valued functions on $[a, b]$ by $C[a, b]$ endowed with the norm $\|f\|_{C}=$ $\sup _{x \in[a, b]}|f(x)|,|$.$| is the absolute value in the real line or the norm in the Banach space. Next we introduce$ a space of $\rho$ - weighted continuous functions in which continuity at $a$ is not stressed. For $0 \leqslant \epsilon<1$, we define

$$
C_{\epsilon, \rho}[a, b]=\left\{f:(a, b] \rightarrow \mathbb{R}:\left(x^{\rho}-a^{\rho}\right)^{\epsilon} f(x) \in C[a, b]\right\}, \quad \rho \neq 0,
$$

endowed with the norm $\|f\|_{C_{\epsilon, \rho}}=\left\|\left(x^{\rho}-a^{\rho}\right)^{\epsilon} f(x)\right\|_{C}$.

$$
C_{\epsilon, \rho}[a, b]=\left\{f:(a, b] \rightarrow \mathbb{R}:(\log x-\log a)^{\epsilon} f(x) \in C[a, b]\right\}, \quad \rho=0,
$$

endowed with the norm $\|f\|_{C_{\epsilon, \rho}}=\|f\|_{C_{\epsilon, \log }}=\left\|(\log x-\log a)^{\epsilon} f(x)\right\|_{C}$. The convention that $C_{0, \rho}[a, b]=$ $C[a, b]$ is used.

Definition 2.1. Let $[a, b]$ be a finite interval, $0 \leqslant \epsilon<1$ and $A C[a, b]$ be the set of absolute continuous functions on $[a, b]$. Then, we define

$$
\begin{aligned}
& A C_{\gamma}^{n}[a, b]=\left\{f:[a, b] \rightarrow C \text { and } \gamma^{n-1} f \in A C[a, b], \gamma=x^{1-\rho} \frac{d}{d x}\right\}, \quad A_{\gamma}^{1}[a, b]=A C[a, b] . \\
& C_{\gamma, \epsilon}^{n}[a, b]=\left\{f:[a, b] \rightarrow C \text { and } \gamma^{n-1} f \in C[a, b], \gamma^{n} f \in C_{\epsilon, \rho}[a, b], \gamma=x^{1-\rho} \frac{d}{d x}\right\},
\end{aligned}
$$

endowed with the norm $\|f\|_{C_{\gamma, e}^{n}}=\sum_{k=0}^{n-1}\left\|\gamma^{k} f\right\|_{C}+\left\|\gamma^{n} f\right\|_{C_{e, \rho}}$. The convention $C_{\gamma, 0}^{n}[a, b]=C_{\gamma}^{n}[a, b]$ endowed with the norm $\|f\|_{C_{\gamma}^{n}}=\sum_{k=0}^{n}\left\|\gamma^{k} f\right\|_{C}$ is used.

Lemma 2.2. Assume $\rho \neq 0$. A function $\mathrm{f} \in \mathrm{AC}_{\gamma}^{\mathrm{n}}[\mathrm{a}, \mathrm{b}]$ if and only if $\mathrm{f}$ is presented in the form

$$
f(x)=\frac{1}{(n-1) !} \int_{a}^{x}\left(\frac{x^{\rho}-t^{\rho}}{\rho}\right)^{n-1} \frac{\left(\gamma^{n} f\right)(t)}{t^{1-\rho}} d t+\sum_{k=0}^{n-1} \frac{\left(\gamma^{k} f\right)(a)}{k !}\left(\frac{x^{\rho}-a^{\rho}}{\rho}\right)^{k} .
$$

Proof. Since $f \in A C_{\gamma}^{n}[a, b]$, from Definition 2.1, $\gamma^{n-1} f \in A C[a, b]$. Hence one can write

$$
\gamma^{n-1} f(x)=\int_{a}^{x} g(t) d t+c_{0}
$$

for some function $g \in \mathrm{L}_{1}[\mathrm{a}, \mathrm{b}]$ and $\mathrm{c}_{0}$ is a constant.

Dividing both sides of (2.2) by $x^{1-\rho}$ and then integrating gives

$$
\gamma^{n-2} f(x)=\int_{a}^{x} \frac{1}{t^{1-\rho}}\left(\int_{a}^{t} g(u) d u+c_{0}\right) d t=\int_{a}^{x}\left(\frac{x^{\rho}-t^{\rho}}{\rho}\right) g(t) d t+c_{0}\left(\frac{x^{\rho}-a^{\rho}}{\rho}\right)+c_{1} .
$$

Dividing both sides of (2.3) by $x^{1-\rho}$ and then integrating once more yields

$$
\gamma^{n-3} f(x)=\int_{a}^{x}\left(\frac{x^{\rho}-t^{\rho}}{\rho}\right)^{2} \frac{g(t)}{2} d t+\frac{c_{0}}{2}\left(\frac{x^{\rho}-a^{\rho}}{\rho}\right)^{2}+c_{1}\left(\frac{x^{\rho}-a^{\rho}}{\rho}\right)+c_{2} .
$$

Repeating the same procedure $n-3$ times, one gets

$$
f(x)=\int_{a}^{x}\left(\frac{x^{\rho}-t^{\rho}}{\rho}\right)^{n-1} \frac{g(t)}{(n-1) !} d t+\sum_{k=0}^{n-1} \frac{c_{k}}{(n-k-1) !}\left(\frac{x^{\rho}-a^{\rho}}{\rho}\right)^{n-k-1} .
$$


It is clear from (2.1) that $\gamma^{n} f(x)=x^{1-\rho} g(x)$ and from the proof that

$$
c_{k}=\gamma^{n} f(a), \quad k=0,1, \cdots, n-1 .
$$

This was the proof of the necessity. To proof the sufficiency it is enough to apply the operator $\gamma^{\mathrm{n}}$ to both sides of (2.1). We should mention that Lemma 2.2 can be adapted to the case of the right integration as

$$
f(x)=\frac{1}{(n-1) !} \int_{x}^{b}\left(\frac{t^{\rho}-x^{\rho}}{\rho}\right)^{n-1} \frac{(-1)^{n}\left(\gamma^{n} f\right)(t)}{t^{1-\rho}} d t+\sum_{k=0}^{n-1} \frac{(-1)^{k}\left(\gamma^{k} f\right)(b)}{k !}\left(\frac{b^{\rho}-x^{\rho}}{\rho}\right)^{k} .
$$

Notice that if we let $\rho \rightarrow 0$ in the representation (2.1) then we get the representation (3.1) in [15] with $\mu=0$. An analogous lemma can be written for the space $C_{\gamma, \epsilon}^{n}[a, b]$ as follows.

Lemma 2.3. Assume $\rho \neq 0$. A function $\mathrm{f} \in \mathrm{C}_{\gamma, \epsilon}^{\mathrm{n}}[\mathrm{a}, \mathrm{b}]$ if and only if $\mathrm{f}$ is presented in the form

$$
f(x)=\frac{1}{(n-1) !} \int_{a}^{x}\left(\frac{x^{\rho}-t^{\rho}}{\rho}\right)^{n-1} g(t) d t+\sum_{k=0}^{n-1} c_{k}\left(\frac{x^{\rho}-a^{\rho}}{\rho}\right)^{k},
$$

where $\mathrm{g}(\mathrm{t}) \in \mathrm{C}_{\epsilon, \rho}[\mathrm{a}, \mathrm{b}]$. Moreover, $\mathrm{g}(\mathrm{x})=\frac{\left(\gamma^{\mathrm{n}} \mathrm{f}\right)(\mathrm{x})}{\mathrm{x}^{1-\rho}}$ and $\mathrm{c}_{\mathrm{k}}=\frac{\left(\gamma^{\mathrm{k}} \mathrm{f}\right)(\mathrm{a})}{\mathrm{k} !}$. In particular, $\mathrm{f} \in \mathrm{C}_{\gamma}^{\mathrm{n}}[\mathrm{a}, \mathrm{b}]$ if and only if $\mathrm{f}$ is presented in the form of $(2.5)$, where $\mathrm{g}(\mathrm{t}) \in \mathrm{C}[\mathrm{a}, \mathrm{b}]$.

Proof. The proof is similar to the proof of Lemma 2.2.

Notice that if we let $\rho \rightarrow 0$ in the representation (2.5) then we get the representation (1.1.29) in [16]. Also, if we let $\rho \rightarrow 1$ in the representations (2.1) and (2.5), respectively, then, we get the representations (1.1.8) and (1.1.23) in [16], respectively.

Now we present a formula for the generalized fractional derivatives of functions in the space $f \in$ $\operatorname{AC}_{\gamma}^{n}[a, b]$.

Theorem 2.4. Let $\operatorname{Re}(\alpha)>0, \mathrm{n}=\operatorname{Re}(\alpha)+1$ and $\mathrm{f} \in \mathrm{AC}_{\gamma}^{\mathrm{n}}[\mathrm{a}, \mathrm{b}]$ or $\mathrm{f} \in \mathrm{C}_{\gamma}^{\mathrm{n}}[\mathrm{a}, \mathrm{b}]$. Then the generalized fractional derivatives of $f$ exist almost everywhere and can be represented in the form

$$
\begin{gathered}
{ }_{a} D^{\alpha, \rho} f(x)=\frac{1}{\Gamma(n-\alpha)} \int_{a}^{x}\left(\frac{x^{\rho}-t^{\rho}}{\rho}\right)^{n-\alpha-1} \frac{\left(\gamma^{n} g\right)(t) d t}{t^{1-\rho}}+\sum_{k=0}^{n-1} \frac{\left(\gamma^{k} f(a)\right)}{\Gamma(k-\alpha+1)}\left(\frac{x^{\rho}-a^{\rho}}{\rho}\right)^{k-\alpha}, \\
D_{b}^{\alpha, \rho} f(x)=\frac{(-1)^{n}}{\Gamma(n-\alpha)} \int_{x}^{b}\left(\frac{t^{\rho}-x^{\rho}}{\rho}\right)^{n-\alpha-1} \frac{\left(\gamma^{n} g\right)(t) d t}{t^{1-\rho}}+\sum_{k=0}^{n-1} \frac{(-1)^{k}\left(\gamma^{k} f(b)\right)}{\Gamma(k-\alpha+1)}\left(\frac{b^{\rho}-x^{\rho}}{\rho}\right)^{k-\alpha} .
\end{gathered}
$$

Proof. Here we prove (2.6). Equation (2.7) can be proved similarly. Apply ${ }_{a} D^{\alpha, \rho}$ to both sides of equation (2.4), then using property (2.11) one gets

$$
\begin{aligned}
{ }_{a} D^{\alpha, \rho} f(x)= & \frac{1}{\Gamma(n-\alpha)(n-1) !} \gamma^{n}\left\{\int_{a}^{x} \int_{a}^{t}\left(\frac{x^{\rho}-t^{\rho}}{\rho}\right)^{n-\alpha-1}\left(\frac{t^{\rho}-u^{\rho}}{\rho}\right)^{n-1} \frac{\left(\gamma^{n} f\right)(u) d u}{u^{1-\rho}} \frac{d t}{t^{1-\rho}}\right\} \\
& +\sum_{k=0}^{n-1} \frac{\left(\gamma^{k} f(a)\right)}{\Gamma(k-\alpha+1)}\left(\frac{x^{\rho}-a^{\rho}}{\rho}\right)^{k-\alpha} .
\end{aligned}
$$

Reversing the order of integration one gets

$$
\begin{aligned}
{ }_{a} D^{\alpha, \rho} f(x)= & \frac{1}{\Gamma(n-\alpha)(n-1) !} \gamma^{n}\left\{\int_{a}^{x} \int_{u}^{x}\left(\frac{x^{\rho}-t^{\rho}}{\rho}\right)^{n-\alpha-1}\left(\frac{t^{\rho}-u^{\rho}}{\rho}\right)^{n-1} \frac{\left(\gamma^{n} f\right)(u) d t}{t^{1-\rho}} \frac{d u}{u^{1-\rho}}\right\} \\
& +\sum_{k=0}^{n-1} \frac{\left(\gamma^{k} f(a)\right)}{\Gamma(k-\alpha+1)}\left(\frac{x^{\rho}-a^{\rho}}{\rho}\right)^{k-\alpha} .
\end{aligned}
$$


Using the change of variable $v=\frac{t^{\rho}-u^{\rho}}{x^{\rho}-u^{\rho}}$, one obtains

$$
\begin{aligned}
{ }_{\mathrm{a}} \mathrm{D}^{\alpha, \rho} \mathrm{f}(\mathrm{x})= & \frac{1}{\Gamma(\mathrm{n}-\alpha)(\mathrm{n}-1) !} \gamma^{\mathrm{n}}\left\{\int_{a}^{x}\left(\frac{x^{\rho}-\mathrm{u}^{\rho}}{\rho}\right)^{2 n-\alpha-1} \frac{\left(\gamma^{\mathrm{n}} \mathrm{f}\right)(\mathrm{u}) \mathrm{du}}{\mathrm{u}^{1-\rho}}\right\} \int_{0}^{1}(1-v)^{n-\alpha-1} v^{\mathrm{n}-1} \mathrm{~d} v \\
& +\sum_{k=0}^{n-1} \frac{\left(\gamma^{\mathrm{k}} \mathrm{f}(\mathrm{a})\right)}{\Gamma(k-\alpha+1)}\left(\frac{x^{\rho}-\mathrm{a}^{\rho}}{\rho}\right)^{\mathrm{k}-\alpha} .
\end{aligned}
$$

Evaluating the second integral in (2.8), one gets

$$
\begin{aligned}
{ }_{a} D^{\alpha, \rho} f(x)= & \frac{\Gamma(n-\alpha) \Gamma(n)}{\Gamma(2 n-\alpha) \Gamma(n-\alpha)(n-1) !} \gamma^{n}\left\{\int_{a}^{x}\left(\frac{x^{\rho}-u^{\rho}}{\rho}\right)^{2 n-\alpha-1} \frac{\left(\gamma^{n} f\right)(u) d u}{u^{1-\rho}}\right\} \\
& +\sum_{k=0}^{n-1} \frac{\left(\gamma^{k} f(a)\right)}{\Gamma(k-\alpha+1)}\left(\frac{x^{\rho}-a^{\rho}}{\rho}\right)^{k-\alpha} .
\end{aligned}
$$

Now applying the operator $\gamma^{\mathrm{n}}$ on the integral, (2.9) becomes

$$
{ }_{a} D^{\alpha, \rho} f(x)=\frac{1}{\Gamma(n-\alpha)} \int_{a}^{x}\left(\frac{x^{\rho}-u^{\rho}}{\rho}\right)^{n-\alpha-1} \frac{\left(\gamma^{n} f\right)(u) d u}{u^{1-\rho}}+\sum_{k=0}^{n-1} \frac{\left(\gamma^{k} f(a)\right)}{\Gamma(k-\alpha+1)}\left(\frac{x^{\rho}-a^{\rho}}{\rho}\right)^{k-\alpha} .
$$

Theorem 2.5. Let $\alpha>\beta>0,1 \leqslant p \leqslant \infty$ and $c \in \mathbb{R}$. Then for $f \in X_{c}^{p}(a, b)$ we have

$$
{ }_{a} D^{\beta, \rho}{ }_{a} I^{\alpha, \rho} f={ }_{a} I^{\alpha-\beta, \rho} f \text {, and } D_{b}^{\beta, \rho} I_{b}^{\alpha, \rho} f=I_{b}^{\alpha-\beta, \rho} f .
$$

Proof. If $\beta=m$ a positive integer, then we have

$$
\begin{aligned}
{ }_{a} D_{a}^{m, \rho} I^{\alpha, \rho} f(x) & =\gamma^{m}\left[\frac{1}{\Gamma(\alpha)} \int_{a}^{x}\left(\frac{x^{\rho}-t^{\rho}}{\rho}\right)^{\alpha-1} \frac{f(t) d t}{t^{1-\rho}}\right] \\
& =\gamma^{m-1}\left[\frac{1}{\Gamma(\alpha-1)} \int_{a}^{x}\left(\frac{x^{\rho}-t^{\rho}}{\rho}\right)^{\alpha-2} \frac{f(t) d t}{t^{1-\rho}}\right] \\
& =\gamma^{m-2}\left[\frac{1}{\Gamma(\alpha-2)} \int_{a}^{x}\left(\frac{x^{\rho}-t^{\rho}}{\rho}\right)^{\alpha-3} \frac{f(t) d t}{t^{1-\rho}}\right] \\
& \vdots \\
& =\frac{1}{\Gamma(\alpha-m)} \int_{a}^{x}\left(\frac{x^{\rho}-t^{\rho}}{\rho}\right)^{\alpha-m-1} \frac{f(t) d t}{t^{1-\rho}} \\
& ={ }_{a} I^{\alpha-m, \rho} f(x) .
\end{aligned}
$$

Now, if $m-1<\beta \leqslant m$ we have

$$
{ }_{a} D^{\beta, \rho}{ }_{a} I^{\alpha, \rho_{f}}=\gamma_{a}^{m} I^{m-\beta, \rho}{ }_{a} I^{\alpha, \rho_{f}}=\gamma^{m}{ }_{a} I^{\alpha+m-\beta, \rho} f={ }_{a} I^{\alpha-\beta, \rho} f .
$$

This was the end of the proof of the first formula. The second can be proved in a similar way.

Theorem 2.6 ([14]). Let $\alpha>0,1 \leqslant p \leqslant \infty$ and $c \in \mathbb{R}$. Then for $f \in X_{c}^{p}(a, b)$ where $a>0, \rho>0$, we have

$$
{ }_{a} D^{\alpha, \rho}{ }_{a} I^{\alpha, \rho} f=f \text {, and } D_{b}^{\alpha, \rho} I_{b}^{\alpha, \rho} f=f .
$$

Theorem 2.7. Let $\operatorname{Re}(\alpha)>0, n=-[-\operatorname{Re}(\alpha)], f \in L(a, b)$ and ${ }_{a} I^{\alpha, \rho} f \in A C_{\gamma}^{n}[a, b]\left(I_{b}^{\alpha, \rho} f \in A C_{\gamma}^{n}[a, b]\right)$. Then

$$
\left({ }_{a} I^{\alpha, \rho}{ }_{a} D^{\alpha, \rho}\right) f(x)=f(x)-\sum_{j=1}^{n} \frac{{ }_{a} D^{\alpha-j, \rho} f(a)}{\Gamma(\alpha-j+1)}\left(\frac{x^{\rho}-a^{\rho}}{\rho}\right)^{\alpha-j},
$$




$$
\left(I_{b}^{\alpha, \rho} D_{b}^{\alpha, \rho}\right) f(x)=f(x)-\sum_{j=1}^{n} \frac{(-1)^{j} D_{b}^{\alpha-j, \rho} f(b)}{\Gamma(\alpha-j+1)}\left(\frac{b^{\rho}-x^{\rho}}{\rho}\right)^{\alpha-j} .
$$

Proof.

$$
\begin{aligned}
\left({ }_{a} I^{\alpha, \rho}{ }_{a} D^{\alpha, \rho}\right) f(x) & =\frac{1}{\Gamma(\alpha)} \int_{a}^{x}\left(\frac{x^{\rho}-t^{\rho}}{\rho}\right)^{\alpha-1}{ }_{a} D^{\alpha, \rho} f(t) \frac{d t}{t^{1-\rho}} \\
& =\frac{1}{\Gamma(\alpha)} \int_{a}^{x}\left(\frac{x^{\rho}-t^{\rho}}{\rho}\right)^{\alpha-1} \gamma^{n}\left({ }_{a} I^{n-\alpha, \rho} f\right)(t) \frac{d t}{t^{1-\rho}} \\
& =\gamma\left[\frac{1}{\Gamma(\alpha+1)} \int_{a}^{x}\left(\frac{x^{\rho}-t^{\rho}}{\rho}\right)^{\alpha} \gamma^{n}\left({ }_{a} I^{n-\alpha, \rho} f\right)(t) \frac{d t}{t^{1-\rho}}\right] \\
& =\gamma\left[\frac{1}{\Gamma(\alpha+1)} \int_{a}^{x}\left(\frac{x^{\rho}-t^{\rho}}{\rho}\right)^{\alpha} \frac{d}{d t}\left(\gamma^{n-1}\left({ }_{a} I^{n-\alpha, \rho} f\right)(t)\right) d t\right] .
\end{aligned}
$$

Now integrating by parts repeatedly leads to

$$
\begin{aligned}
\left({ }_{a} I^{\alpha, \rho}{ }_{a} D^{\alpha, \rho}\right) f(x)= & \gamma\left[\frac{1}{\Gamma(\alpha-n+1)} \int_{a}^{x}\left(\frac{x^{\rho}-t^{\rho}}{\rho}\right)^{\alpha-n}{ }_{a} I^{n-\alpha, \rho} f(t) \frac{d t}{t^{1-\rho}}\right. \\
& \left.-\sum_{j=1}^{n} \frac{\left(\gamma^{n-j}\left(a I^{n-\alpha, \rho}\right)\right) f(a)}{\Gamma(\alpha+2-j)}\left(\frac{x^{\rho}-a^{\rho}}{\rho}\right)^{\alpha-j+1}\right] \\
= & \gamma\left[{ }_{a} I^{\alpha-n+1, \rho}{ }_{a} I^{n-\alpha, \rho} f(x)-\sum_{j=1}^{n} \frac{\left(\gamma^{n-j}\left({ }_{a} I^{n-\alpha, \rho} f\right)\right)(a)}{\Gamma(\alpha+2-j)}\left(\frac{x^{\rho}-a^{\rho}}{\rho}\right)^{\alpha-j+1}\right] .
\end{aligned}
$$

By using the semigroup property (Theorem 4.1.) in [13], one obtains

$$
\left({ }_{a} I^{\alpha, \rho}{ }_{a} D^{\alpha, \rho}\right) f(x)=\gamma\left[{ }_{a} I^{1, \rho} f(x)-\sum_{j=1}^{n} \frac{{ }_{a} D^{\alpha-j, \rho} f(a)}{\Gamma(\alpha+2-j)}\left(\frac{x^{\rho}-a^{\rho}}{\rho}\right)^{\alpha-j+1}\right] .
$$

The result is reached after applying the operator $\gamma$ to the integral. (2.10) is proved analogously

Lemma 2.8. Let $\operatorname{Re}(\alpha) \geqslant 0$ and $\operatorname{Re}(\beta)>0$. Then,

$$
\begin{gathered}
\left({ }_{a} D^{\alpha, \rho}\left(\frac{t^{\rho}-a^{\rho}}{\rho}\right)^{\beta-1}\right)(x)=\frac{\Gamma(\beta)}{\Gamma(\beta-\alpha)}\left(\frac{x^{\rho}-a^{\rho}}{\rho}\right)^{\beta-\alpha-1}, \\
\left(D_{b}^{\alpha, \rho}\left(\frac{b^{\rho}-t^{\rho}}{\rho}\right)^{\beta-1}\right)(x)=\frac{\Gamma(\beta)}{\Gamma(\beta-\alpha)}\left(\frac{b^{\rho}-x^{\rho}}{\rho}\right)^{\beta-\alpha-1}, \\
\left({ }_{a} D^{\alpha, \rho}\left(\frac{t^{\rho}-a^{\rho}}{\rho}\right)^{\alpha-i}\right)(x)=0, \quad i=1,2, \cdots,[\operatorname{Re}(\alpha)]+1, \\
\left(D_{b}^{\alpha, \rho}\left(\frac{b^{\rho}-t^{\rho}}{\rho}\right)^{\alpha-i}\right)(x)=0, \quad i=1,2, \cdots,[\operatorname{Re}(\alpha)]+1 .
\end{gathered}
$$

Proof. Here we prove (2.11) and (2.12). The rest of the results are proved analogously.

$$
\begin{aligned}
& { }_{a} D^{\alpha, \rho}\left(\frac{t^{\rho}-a^{\rho}}{\rho}\right)^{\beta-1}(x) \\
& =\frac{1}{\Gamma(n-\alpha)}\left(x^{1-\rho} \frac{d}{d x}\right)^{n}\left[\int_{a}^{x}\left(\frac{x^{\rho}-t^{\rho}}{\rho}\right)^{n-\alpha-1}\left(\frac{t^{\rho}-a^{\rho}}{\rho}\right)^{\beta-1} \frac{d t}{t^{1-\rho}}\right]
\end{aligned}
$$




$$
\begin{aligned}
& \left.=\frac{1}{\Gamma(n-\alpha)}\left(x^{1-\rho} \frac{d}{d x}\right)^{n}\left[\left(\frac{x^{\rho}-a^{\rho}}{\rho}\right)^{n-\alpha+\beta-1} \int_{0}^{1}(1-u)^{n-\alpha-1} u^{\beta-1} d u\right], \quad \text { (where } u=\frac{t^{\rho}-a^{\rho}}{x^{\rho}-a^{\rho}}\right) \\
& =\frac{\Gamma(\beta) \Gamma(n-\alpha)}{\Gamma(\beta+n-\alpha) \Gamma(n-\alpha)}\left(x^{1-\rho} \frac{d}{d x}\right)^{n}\left[\left(\frac{x^{\rho}-a^{\rho}}{\rho}\right)^{n-\alpha+\beta-1}\right] \quad \text { (using properties of the beta function) } \\
& =\frac{\Gamma(\beta)}{\Gamma(\beta+n-\alpha)} \frac{\Gamma(\beta+n-\alpha)}{\Gamma(\beta-\alpha)}\left(\frac{x^{\rho}-a^{\rho}}{\rho}\right)^{\beta-\alpha-1}=\frac{\Gamma(\beta)}{\Gamma(\beta-\alpha)}\left(\frac{x^{\rho}-a^{\rho}}{\rho}\right)^{\beta-\alpha-1} .
\end{aligned}
$$

This was the end of the proof of (2.11). Now,

$$
\begin{aligned}
& { }_{a} D^{\alpha, \rho}\left(\frac{t^{\rho}-a^{\rho}}{\rho}\right)^{\alpha-i}(x)=\frac{1}{\Gamma(n-\alpha)}\left(x^{1-\rho} \frac{d}{d x}\right)^{n}\left[\int_{a}^{x}\left(\frac{x^{\rho}-t^{\rho}}{\rho}\right)^{n-\alpha-1}\left(\frac{t^{\rho}-a^{\rho}}{\rho}\right)^{\alpha-i} \frac{d t}{t^{1-\rho}}\right] \\
& =\frac{1}{\Gamma(n-\alpha)}\left(x^{1-\rho} \frac{d}{d x}\right)^{n}\left[\left(\frac{x^{\rho}-a^{\rho}}{\rho}\right)^{n-i} \int_{0}^{1}(1-u)^{n-\alpha-1} u^{\alpha-i} d u\right. \\
& =\frac{\Gamma(\alpha-i+1) \Gamma(n-\alpha)}{\Gamma(n-i+1) \Gamma(n-\alpha)}\left(x^{1-\rho} \frac{d}{d x}\right)^{n}\left[\left(\frac{x^{\rho}-a^{\rho}}{\rho}\right)^{n-i}\right]=0 .
\end{aligned}
$$

The limiting case $\rho \rightarrow 0$ in Lemma 2.8 will lead to the Hadamard fractional formulas with $\left(\frac{x^{\rho}-a^{\rho}}{\rho}\right)$ replaced with $\ln (x / a)$ and $\left(\frac{b^{\rho}-x^{\rho}}{\rho}\right)$ replaced with $\ln (b / x)$. Also, the case $\rho \rightarrow 1$ will result in the RiemannLiouville's formulas.

\section{Caputo modification of the generalized fractional derivative}

Below we present the definition of the generalized Caputo fractional derivative of any order which is different from the definition stated in [13].

Definition 3.1. Let $\operatorname{Re}(\alpha) \geqslant 0$ and $n=[\operatorname{Re}(\alpha)]+1$. If $f \in A C_{\gamma}^{n}[a, b]$, where $0<a<b<\infty$, we define the left and right generalized Caputo fractional derivatives of $f$ of order $\alpha$ by

$$
\begin{aligned}
& { }_{a}^{C} D^{\alpha, \rho} f(x)={ }_{a} D^{\alpha, \rho}\left[f(t)-\sum_{k=0}^{n-1} \frac{\gamma^{k} f(a)}{k !}\left(\frac{t^{\rho}-a^{\rho}}{\rho}\right)^{k}\right](x), \\
& { }^{C} D_{b}^{\alpha, \rho} f(x)=D_{b}^{\alpha, \rho}\left[f(t)-\sum_{k=0}^{n-1} \frac{(-1)^{k} \gamma^{k} f(b)}{k !}\left(\frac{b^{\rho}-t^{\rho}}{\rho}\right)^{k}\right](x),
\end{aligned}
$$

respectively. In case $0<\operatorname{Re}(\alpha)<1$, we have

$$
{ }_{a}^{C} D^{\alpha, \rho} f(x)={ }_{a} D^{\alpha, \rho}[f(t)-f(a)](x),
$$

and

$$
{ }^{C} D_{b}^{\alpha, \rho} f(x)=D_{b}^{\alpha, \rho}[f(t)-f(b)](x) .
$$

Theorem 3.2. Let $\operatorname{Re}(\alpha) \geqslant 0, \mathrm{n}=[\operatorname{Re}(\alpha)]+1$ and $\mathrm{f} \in \mathrm{AC}_{\gamma}^{\mathrm{n}}[\mathrm{a}, \mathrm{b}]$, where $0<\mathrm{a}<\mathrm{b}<\infty$. Then,

1. If $\alpha \notin \mathbb{N}_{0}$,

$$
\begin{aligned}
& { }_{a}^{C} D^{\alpha, \rho} f(x)=\frac{1}{\Gamma(n-\alpha)} \int_{a}^{x}\left(\frac{x^{\rho}-t^{\rho}}{\rho}\right)^{n-\alpha-1} \frac{\left(\gamma^{n} f\right)(t) d t}{t^{1-\rho}}={ }_{a} I^{n-\alpha, \rho}\left(\gamma^{n} f\right)(x), \\
& { }^{C} D_{b}^{\alpha, \rho} f(x)=\frac{1}{\Gamma(n-\alpha)} \int_{x}^{b}\left(\frac{t^{\rho}-x^{\rho}}{\rho}\right)^{n-\alpha-1} \frac{(-1)^{n}\left(\gamma^{n} f\right)(t) d t}{t^{1-\rho}}=I_{b}^{n-\alpha, \rho}\left(\gamma^{n} f\right)(x) .
\end{aligned}
$$


2. If $\alpha \in \mathbb{N}$

$$
{ }_{a}^{C} D^{\alpha, \rho} f=\gamma^{n} f, \quad{ }^{C} D_{b}^{\alpha, \rho} f=(-1)^{n} \gamma^{n} f .
$$

Particularly,

$$
{ }_{\mathrm{a}}^{\mathrm{C}} \mathrm{D}^{0, \rho} \mathrm{f}=\mathrm{f}, \quad \mathrm{C}_{\mathrm{b}} \mathrm{D}_{\mathrm{b}}^{0, \rho_{f}}=\mathrm{f} .
$$

Proof. (3.2) and (3.3) are consequences of Theorem 2.4. Now, when $\alpha=n$ we have

$$
f(x)={ }_{a} I^{n, \rho}\left(\gamma^{n} f\right)(x)+\sum_{k=0}^{n-1} \frac{\gamma^{k} f(a)}{k !}\left(\frac{t^{\rho}-a^{\rho}}{\rho}\right)^{k} .
$$

From Lemma 2.2, one gets ${ }_{a}^{C} D^{\alpha, \rho} f=\gamma^{n} f$. The second part of (3.4) can be proved likewise.

Theorem 3.3. Let $\operatorname{Re}(\alpha) \geqslant 0, \mathrm{n}=[\operatorname{Re}(\alpha)]+1$ and $\mathrm{f} \in \mathrm{C}_{\gamma}^{\mathrm{n}}[\mathrm{a}, \mathrm{b}]$, where $0<\mathrm{a}<\mathrm{b}<\infty$. Then, ${ }_{\mathrm{a}}^{\mathrm{C}} \mathrm{D}^{\alpha, \rho} \mathrm{f}$ and ${ }^{C} D_{b}^{\alpha, \rho}$ f can be represented as in (3.2) and (3.3), respectively if $\alpha \notin \mathbb{N}_{0}$. If $\alpha \in \mathbb{N}$, (3.4) holds. Moreover ${ }_{a}^{C} D^{\alpha, \rho} f$ and ${ }^{C} \mathrm{D}_{\mathrm{b}}^{\alpha, \rho} \mathrm{f}$ are continuous on $[\mathrm{a}, \mathrm{b}]$ and

$$
{ }_{a}^{C} D^{\alpha, \rho} f(a)=0, \quad \quad{ }^{C} D_{b}^{\alpha, \rho} f(b)=0 .
$$

Proof. The representation of ${ }_{a}^{C} D^{\alpha, \rho_{f}}$ and ${ }^{C} D_{b}^{\alpha, \rho} f$ can be proved as in the proof of Theorem 2.4. Now, since

$$
{ }_{a}^{c} D^{\alpha, \rho} f(x)=\frac{1}{\Gamma(n-\alpha)} \int_{a}^{x}\left(\frac{x^{\rho}-t^{\rho}}{\rho}\right)^{n-\alpha-1} \frac{\left(\gamma^{n} f\right)(t) d t}{t^{1-\rho}},
$$

we have

$$
\left\|{ }_{a}^{C} D^{\alpha, \rho} f\right\|_{C} \leqslant \frac{\left\|\gamma^{n} f\right\|_{C}}{|\Gamma(n-\alpha)(n-\operatorname{Re}(\alpha))|}\left|\frac{b^{\rho}-a^{\rho}}{\rho}\right|^{n-\operatorname{Re}(\alpha)} .
$$

Thus the continuity is proved. The identities in (3.5) hold since

$$
\left|{ }_{a}^{C} D^{\alpha, \rho} f\right| \leqslant \frac{\left\|\gamma^{n} f\right\|_{C}}{|\Gamma(n-\alpha)(n-\operatorname{Re}(\alpha))|}\left|\frac{\chi^{\rho}-a^{\rho}}{\rho}\right|^{n-\operatorname{Re}(\alpha)},
$$

and

$$
\left|{ }^{C} D_{b}^{\alpha, \rho} f\right| \leqslant \frac{\left\|\gamma^{n} f\right\|_{C}}{|\Gamma(n-\alpha)(n-\operatorname{Re}(\alpha))|}\left|\frac{b^{\rho}-x^{\rho}}{\rho}\right|^{n-\operatorname{Re}(\alpha)} .
$$

Theorem 3.4. Let $\operatorname{Re}(\alpha) \geqslant 0, \mathrm{n}=[\operatorname{Re}(\alpha)]+1$. If $\alpha \notin \mathbb{N},{ }_{\mathrm{a}}^{\mathrm{C}} \mathrm{D}^{\alpha, \rho}$ is bounded from the space $\mathrm{C}_{\gamma}^{\mathrm{n}}[\mathrm{a}, \mathrm{b}]$ to the space $C_{a}[a, b]=\{g \in C[a, b]: g(a)=0\}$ and ${ }^{C} D_{b}^{\alpha, \rho}$ is bounded from the the space $C_{\gamma}^{n}[a, b]$ to the space $\mathrm{C}_{\mathrm{b}}[\mathrm{a}, \mathrm{b}]=\{\mathrm{g} \in \mathrm{C}[\mathrm{a}, \mathrm{b}]: \mathrm{g}(\mathrm{b})=0\}$ and

$$
\left\|{ }_{a}^{C} D^{\alpha, \rho} f\right\|_{C_{a}} \leqslant \frac{\left\|\gamma^{n} f\right\|_{C_{\gamma}^{n}}}{|\Gamma(n-\alpha)(n-\operatorname{Re}(\alpha))|}\left|\frac{b^{\rho}-a^{\rho}}{\rho}\right|^{n-\operatorname{Re}(\alpha)},
$$

and

$$
\left\|{ }^{\mathrm{C}} \mathrm{D}_{\mathrm{b}}^{\alpha, \rho} \mathrm{f}\right\|_{\mathrm{C}_{\mathrm{b}}} \leqslant \frac{\left\|\gamma^{\mathrm{n}} \mathrm{f}\right\|_{\mathrm{C}_{\gamma}^{n}}}{|\Gamma(\mathrm{n}-\alpha)(\mathrm{n}-\operatorname{Re}(\alpha))|}\left|\frac{\mathrm{b}^{\rho}-\mathrm{a}^{\rho}}{\rho}\right|^{\mathrm{n}-\operatorname{Re}(\alpha)} .
$$

If $\alpha \in \mathbb{N},{ }_{\mathrm{a}}^{\mathrm{C}} \mathrm{D}^{\alpha, \rho}$ and ${ }^{\mathrm{C}} \mathrm{D}_{\mathrm{b}}^{\alpha, \rho}$ are bounded from the space $\mathrm{C}_{\gamma}^{\mathrm{n}}[\mathrm{a}, \mathrm{b}]$ to the space $\mathrm{C}[\mathrm{a}, \mathrm{b}]$ and

$$
\left\|{ }_{a}^{C} D^{n, \rho} f\right\|_{C} \leqslant\|f\|_{C_{\gamma}^{n},}, \quad\left\|{ }^{C} D_{b}^{n, \rho} f\right\|_{C} \leqslant\|f\|_{C_{\gamma}^{n}} .
$$

Proof. Equations (3.8) and (3.9) follow from (3.6) and (3.7), respectively. The inequalities in (3.10) are straightforward. 
Below we state the inverse properties.

Theorem 3.5. Let $\operatorname{Re}(\alpha) \geqslant 0, \mathrm{n}=[\operatorname{Re}(\alpha)]+1$ and $\mathrm{f} \in \mathrm{C}[\mathrm{a}, \mathrm{b}]$.

1. If $\operatorname{Re}(\alpha) \neq 0$ or $\alpha \in \mathbb{N}$, then

$$
{ }_{a}^{C} D^{\alpha, \rho}{ }_{a} I^{\alpha, \rho} f(x)=f(x), \quad{ }^{C} D_{b}^{\alpha, \rho} I_{b}^{\alpha, \rho} f(x)=f(x) .
$$

2. If $\operatorname{Re}(\alpha) \neq 0$ and $\operatorname{Re}(\alpha) \in \mathbb{N}$, then

$$
\begin{gathered}
{ }_{a}^{C} D^{\alpha, \rho}{ }_{a} I^{\alpha, \rho} f(x)=f(x)-\frac{{ }_{a} I^{\alpha+1-n, \rho} f(a)}{\Gamma(n-\alpha)}\left(\frac{x^{\rho}-a^{\rho}}{\rho}\right)^{n-\alpha}, \\
{ }^{C} D_{b}^{\alpha, \rho} I_{b}^{\alpha, \rho} f(x)=f(x)-\frac{I_{b}^{\alpha+1-n, \rho} f(b)}{\Gamma(n-\alpha)}\left(\frac{b^{\rho}-x^{\rho}}{\rho}\right)^{n-\alpha} .
\end{gathered}
$$

Proof. From (3.1), one has

$$
{ }_{a}^{C} D^{\alpha, \rho}{ }_{a} I^{\alpha, \rho} f(x)={ }_{a} D_{a}^{\alpha, \rho} I^{\alpha, \rho} f(x)-\sum_{k=0}^{n-1} \frac{\gamma^{k}\left({ }_{a} I^{\alpha, \rho} f\right)(a)}{\Gamma(k-\alpha+1)}\left(\frac{x^{\rho}-a^{\rho}}{\rho}\right)^{k} .
$$

From Theorem 2.7 and Theorem 3.2 one has $\gamma^{k}\left({ }_{a} I^{\alpha, \rho}\right) f={ }_{a} I^{\alpha-k, \rho} f$ and ${ }_{a} D^{\alpha, \rho}{ }_{a} I^{\alpha, \rho} f=f$, respectively. Thus (3.14) reads

$$
{ }_{a}^{C} D^{\alpha, \rho}{ }_{a} I^{\alpha, \rho} f(x)=f(x)-\sum_{k=0}^{n-1} \frac{\left({ }_{a} I^{\alpha-k, \rho}\right) f(a)}{\Gamma(k-\alpha+1)}\left(\frac{x^{\rho}-a^{\rho}}{\rho}\right)^{k} .
$$

On the other hand, it should be easy to verify that $\left({ }_{a} I^{\alpha-k, \rho}\right) f(a)=0$, because of the following estimate

$$
\left|{ }_{a} I^{\alpha-k, \rho} f(x)\right| \leqslant \frac{\|f\|_{C}}{|\Gamma(\alpha-k)(\operatorname{Re}(\alpha)-k)|}\left(\frac{x^{\rho}-a^{\rho}}{\rho}\right)^{\operatorname{Re}(\alpha)-k} .
$$

This is the end of the proof of the first identity in (3.11). The second identity is proved in a similar way.

Now if $\alpha=m+i \beta, \beta \neq 0$, then we have $n=m+1 \geqslant 2$ and $\gamma^{k}\left(a_{a} I^{\alpha, \rho}\right) f={ }_{a} I^{\alpha-k, \rho} f$ is valid when $k=0,1,2, \cdots, m-1$. Because of the estimate (3.16) we have $\left({ }_{a} I^{\alpha-k, \rho}\right) f(a)=0, k=0,1,2, \cdots, m-1$. Substituting in (3.15), we get (3.12). (3.13) is proved similarly.

Theorem 3.6. Let $\mathrm{f} \in \mathrm{AC}_{\gamma}^{\mathrm{n}}[\mathrm{a}, \mathrm{b}]$ or $\mathrm{C}_{\gamma}^{\mathrm{n}}[\mathrm{a}, \mathrm{b}]$ and $\alpha \in \mathbb{C}$. Then

$$
\begin{aligned}
& { }_{a} I^{\alpha, \rho} \underset{a}{C} D^{\alpha, \rho} f(x)=f(x)-\sum_{k=0}^{n-1} \frac{\left(\gamma^{k} f\right)(a)}{k !}\left(\frac{x^{\rho}-a^{\rho}}{\rho}\right)^{k}, \\
& I_{b}^{\alpha, \rho}{ }^{C} D_{b}^{\alpha, \rho} f(x)=f(x)-\sum_{k=0}^{n-1} \frac{(-1)^{k}\left(\gamma^{k} f\right)(b)}{k !}\left(\frac{b^{\rho}-x^{\rho}}{\rho}\right)^{k} .
\end{aligned}
$$

In particular, if $0<\alpha \leqslant 1$, we have

$$
\begin{gathered}
{ }_{a} I^{\alpha, \rho}{ }_{a}^{C} D^{\alpha, \rho} f(x)=f(x)-f(a), \\
I_{b}^{\alpha, \rho}{ }^{C} D_{b}^{\alpha, \rho} f(x)=f(x)-f(b) .
\end{gathered}
$$

Proof. The proof is done by using the semigroup property [13, Theorem 4.1] and Theorem $2.7(\alpha=n)$

$$
{ }_{a} I^{\alpha, \rho} \underset{a}{C} D^{\alpha, \rho} f(x)={ }_{a} I^{\alpha, \rho}{ }_{a} I^{n-\alpha, \rho} \gamma^{n} f={ }_{a} I^{n, \rho} \gamma^{n} f(x)-\sum_{k=0}^{n-1} \frac{\left(\gamma^{k} f\right)(a)}{k !}\left(\frac{x^{\rho}-a^{\rho}}{\rho}\right)^{k} .
$$

Equation (3.17) can be proved analogously. 
Next we present the composition rule for two generalized Caputo fractional derivatives.

Theorem 3.7. Let $f \in \operatorname{AC}_{\gamma}^{m+n}[a, b], 0<a<b<\infty, \alpha \geqslant 0$ and $\beta \geqslant 0$ such that $n-1<\alpha \leqslant n$ and $\mathrm{m}-1<\beta \leqslant \mathrm{m}$. Then

$$
{ }_{a}^{C} D^{\alpha, \rho}{ }_{a}^{C} D^{\beta, \rho} f(x)={ }_{a}^{C} D^{\alpha+\beta, \rho} f(x) .
$$

Proof. Let us assume that $m \geqslant n$. Thus $m=n+k, k=0,1,2, \cdots, m-n$. Then the proof can be done by using Theorem 2.7, Theorem 3.2 and [13, Theorem 4.1]. In fact,

$$
\begin{aligned}
{ }_{a}^{C} D^{\alpha, \rho}{ }_{a}^{C} D^{\beta, \rho} f(x) & ={ }_{a} I^{n-\alpha, \rho} \gamma_{a}^{n} I^{m-\beta, \rho} \gamma^{m} f(x) \\
& ={ }_{a} I^{n-\alpha, \rho} \gamma^{n}{ }_{a} I^{n+k-\beta, \rho} \gamma^{n+k} f(x) \\
& ={ }_{a} I^{n-\alpha, \rho} \gamma_{a}^{n}{ }_{a} I^{n-\beta, \rho}{ }_{a} I^{k, \rho} \gamma^{n+k} f(x) \\
& ={ }_{a} I^{n-\alpha, \rho}{ }_{a} D^{\beta, \rho}{ }_{a} I^{k, \rho} \gamma^{n+k} f(x) \\
& ={ }_{a} I^{n-\alpha-\beta, \rho}{ }_{a} I^{\beta, \rho}{ }_{a} D^{\beta, \rho}{ }_{a} I^{k, \rho} \gamma^{n+k} f(x) \gamma^{n+k} f(x) \\
& ={ }_{a} I^{n-\alpha-\beta, \rho}\left[{ }_{a} I^{k, \rho} \gamma^{n+k} f(x)-\sum_{j=1}^{n} \frac{\gamma^{n-j}\left({ }_{a} I_{a}^{n-\beta, \rho} I^{k, \rho} \gamma^{n+k} f(a)\right)}{\Gamma(\beta-j+1)}\left(\frac{x^{\rho}-a^{\rho}}{\rho}\right)^{j}\right] \\
& ={ }_{a} I^{n-\alpha-\beta, \rho}\left[{ }_{a} I^{k, \rho} \gamma^{n+k} f(x)-\sum_{j=1}^{n} \frac{\gamma^{n-j}\left({ }_{a}^{C} D^{\beta, \rho} f(a)\right)}{\Gamma(\beta-j+1)}\left(\frac{x^{\rho}-a^{\rho}}{\rho}\right)^{j}\right] \\
& ={ }_{a} I^{n-\alpha-\beta, \rho}\left[{ }_{a} I^{k, \rho} \gamma^{n+k} f(x)-0\right] \\
& ={ }_{a} I^{n+k-\alpha-\beta, \rho} \gamma^{n+k} f(x) \\
& ={ }_{a}^{C} D^{\alpha+\beta, \rho} f(x) .
\end{aligned}
$$

Equation (3.17) is proved using similar arguments.

Lemma 3.8. Let $\operatorname{Re}(\alpha)>0, \mathrm{n}=[\operatorname{Re}(\alpha)+1]+1$ and $\operatorname{Re}(\beta)>0$. Then

$$
\begin{array}{ll}
{ }_{\mathrm{a}}^{C} D^{\alpha, \rho}\left(\frac{t^{\rho}-a^{\rho}}{\rho}\right)^{\beta-1}(x)=\frac{\Gamma(\beta)}{\Gamma(\beta-\alpha)}\left(\frac{x^{\rho}-a^{\rho}}{\rho}\right)^{\beta-\alpha-1}, \quad \operatorname{Re}(\beta)>n, \\
{ }^{C} D_{b}^{\alpha, \rho}\left(\frac{b^{\rho}-t^{\rho}}{\rho}\right)^{\beta-1}(x)=\frac{\Gamma(\beta)}{\Gamma(\beta-\alpha)}\left(\frac{b^{\rho}-x^{\rho}}{\rho}\right)^{\beta-\alpha-1}, \quad \operatorname{Re}(\beta)>n .
\end{array}
$$

Proof.

$$
\begin{aligned}
{ }_{a}^{C} D^{\alpha, \rho} & \left(\frac{t^{\rho}-a^{\rho}}{\rho}\right)^{\beta-1}(x)=\frac{1}{\Gamma(n-\alpha)} \int_{a}^{x}\left(\frac{x^{\rho}-t^{\rho}}{\rho}\right)^{n-\alpha-1}\left[\left(t^{1-\rho} \frac{d}{d t}\right)^{n}\left(\frac{t^{\rho}-a^{\rho}}{\rho}\right)^{\beta-1}\right] \frac{d t}{t^{1-\rho}} \\
& =\frac{\Gamma(\beta)}{\Gamma(\beta-n) \Gamma(n-\alpha)} \int_{a}^{x}\left(\frac{x^{\rho}-t^{\rho}}{\rho}\right)^{n-\alpha-1}\left(\frac{t^{\rho}-a^{\rho}}{\rho}\right)^{\beta-n-1} \frac{d t}{t^{1-\rho}} \quad \text { (differentiation inside the integral) } \\
& =\frac{\Gamma(\beta)}{\Gamma(\beta-n) \Gamma(n-\alpha)}\left(\frac{x^{\rho}-a^{\rho}}{\rho}\right)^{\beta-\alpha-1} \int_{0}^{1}(1-u)^{n-\alpha-1} u^{\beta-n-1} d u, \quad u=\frac{t^{\rho}-a^{\rho}}{x^{\rho}-a^{\rho}} \\
& =\frac{\Gamma(\beta)}{\Gamma(\beta-n) \Gamma(n-\alpha)}\left(\frac{x^{\rho}-a^{\rho}}{\rho}\right)^{\beta-\alpha-1} \frac{\Gamma(n-\alpha) \Gamma(\beta-n)}{\Gamma(\beta-\alpha)} \quad \text { (using properties of the beta function) } \\
& =\frac{\Gamma(\beta)}{\Gamma(\beta-\alpha)}\left(\frac{x^{\rho}-a^{\rho}}{\rho}\right)^{\beta-\alpha-1} .
\end{aligned}
$$

Equation (3.18) can be proved likewise.

Lemma 3.9.

$$
{ }_{a}^{C} D^{\alpha, \rho}\left(\frac{x^{\rho}-a^{\rho}}{\rho}\right)^{k}=0, \quad{ }^{C} D_{b}^{\alpha, \rho}\left(\frac{b^{\rho}-x^{\rho}}{\rho}\right)^{k}(x)=0, \quad k=0,1,2, \cdots, n-1 .
$$


Particularly

$$
{ }_{\mathrm{a}}^{\mathrm{C}} \mathrm{D}^{\alpha, \rho}(1)=0, \quad{ }^{\mathrm{C}} \mathrm{D}_{\mathrm{b}}^{\alpha, \rho}(1)=0 .
$$

Proof.

$$
{ }_{a}^{C} D^{\alpha, \rho}\left(\frac{t^{\rho}-a^{\rho}}{\rho}\right)^{k}(x)={ }_{a} I^{n-\alpha, \rho}\left[\left(t^{1-\rho} \frac{d}{d t}\right)^{n}\left(\frac{t^{\rho}-a^{\rho}}{\rho}\right)^{k}\right](x)={ }_{a} I^{n-\alpha, \rho}[0](x)=0 .
$$

The rest formulas can be proved similarly.

From Definition 3.1 and Lemma 3.8, we can set the following relation between generalized Caputo and Riemann derivatives:

Theorem 3.10. For and $\alpha>0$ and $\rho \neq 0$, we have

$$
{ }_{a}^{C} D^{\alpha, \rho} f(x)={ }_{a} D^{\alpha, \rho} f(x)-\sum_{k=0}^{n-1} \frac{\gamma^{k} f(a)}{\Gamma(k+1-\alpha)}\left(\frac{x^{\rho}-a^{\rho}}{\rho}\right)^{k-\alpha},
$$

and

$$
{ }^{C} D_{b}^{\alpha, \rho} f(x)=D_{b}^{\alpha, \rho} f(x)-\sum_{k=0}^{n-1} \frac{\left(-1^{k}\right) \gamma^{k} f(b)}{\Gamma(k+1-\alpha)}\left(\frac{b^{\rho}-x^{\rho}}{\rho}\right)^{k-\alpha} .
$$

At last, we give the relation between the generalized Caputo fractional derivatives and the known ones.

Theorem 3.11. Let $\alpha \in \mathbb{C}, \operatorname{Re}(\alpha)>0$ and $n=[\operatorname{Re}(\alpha)]+1$. Then

1.

$$
\lim _{\rho \rightarrow 1}{ }_{a}^{C} D^{\alpha, \rho} f(x)=\frac{1}{\Gamma(n-\alpha)} \int_{a}^{x}(x-t)^{n-\alpha-1} f^{(n)}(t) d t={ }_{a}^{c} D^{\alpha} f(x) .
$$

2.

$$
\lim _{\rho \rightarrow 1}{ }^{C} D_{b}^{\alpha, \rho} f(x)=\frac{1}{\Gamma(n-\alpha)} \int_{a}^{x}(x-t)^{n-\alpha-1}(-1)^{n} f^{(n)}(t) d t={ }^{C} D_{b}^{\alpha} f(x)
$$

3.

$$
\lim _{\rho \rightarrow 0}{ }_{a}^{C} D^{\alpha, \rho} f(x)=\frac{1}{\Gamma(n-\alpha)} \int_{a}^{x}(\log x-\log t)^{n-\alpha-1}\left(\left(t \frac{d}{d t}\right)^{n} f\right)(t) \frac{d t}{t}={ }_{a}^{C} \mathcal{D}^{\alpha} f(x) .
$$

4.

$$
\lim _{\rho \rightarrow 0}{ }^{C} D_{b}^{\alpha, \rho} f(x)=\frac{1}{\Gamma(n-\alpha)} \int_{a}^{x}(\log t-\log x)^{n-\alpha-1}(-1)^{n}\left(\left(-t \frac{d}{d t}\right)^{n} f\right)(t) \frac{d t}{t}={ }^{C} \mathcal{D}_{b}^{\alpha} f(x) .
$$

Proof. The limits in (3.19) and (3.20) are evaluated replacing $\rho$ by 0 directly. While, the limits in (3.20) and (3.21) are evaluated by using the L'Hôspital rule.

It should be noted that the derivatives on the right hand sides in (3.19) and (3.20) are respectively the left and right Caputo derivatives [16, 20]. While, the derivatives on the right hand sides in (3.21) and (3.22) are respectively the left and right Caputo-Hadamard derivatives developed in [8] and [12].

\section{Conclusions}

The fundamental issue of the fractional operators and their generalized versions is to define them correctly in the right space of functions. In this paper, we defined the generalized fractional derivatives of functions in the spaces of absolutely differentiable continuous and differentiable continuous functions. Since Caputo derivatives describe better some physical problems involving memory effect, we defined the Caputo version of the generalized fractional derivatives. We believe that this Caputo 
version of the generalized fractional derivative would be useful for researchers working on modeling real world phenomena described by fractional operators. Finally, we noticed that the limiting case as $\rho \rightarrow 0$ leads to the Hadamard and Caputo-Hadamard results by noting that $\lim _{\rho \rightarrow 0}\left(\frac{x^{\rho}-a^{\rho}}{\rho}\right)=\ln \left(\frac{x}{a}\right)$ and $\lim _{\rho \rightarrow 0}\left(\frac{\mathrm{b}^{\rho}-\mathrm{x}^{\rho}}{\rho}\right)=\ln \left(\frac{\mathrm{b}}{\mathrm{x}}\right)$. Also, the case $\rho=1$ will result in Riemann-Liouville's and Caputo fractional derivatives.

\section{References}

[1] T. Abdeljawad, Dual identities in fractional difference calculus within Riemann, Adv. Difference Equ., 2013 (2013), 16 pages. 1

[2] T. Abdeljawad, On delta and nabla Caputo fractional differences and dual identities, Discrete Dyn. Nat. Soc., 2013 (2013), 12 pages.

[3] T. Abdeljawad, D. Baleanu, Fractional differences and integration by parts, J. Comput. Anal. Appl., 13 (2011), 574-582.

[4] T. Abdeljawad, D. Baleanu, Discrete fractional differences with nonsingular discrete Mittag-Leffler kernels, Adv. Difference Equ., 2016 (2016), 18 pages. 1

[5] R. Almeida, A. B. Malinowska, T. Odzijewicz, Fractional differential equations with dependence on the CaputoKatugampola derivative, J. Comput. Nonlinear Dyn., 11 (2016), 11 pages. 1

[6] F. M. Atıcı, S. Şengül, Modeling with fractional difference equations, J. Math. Anal. Appl., 369 (2010), 1-9. 1

[7] M. Caputo, M. Fabrizio, A new definition of fractional derivative without singular kernel, Progr. Fract. Differ. Appl., 1 (2015), 73-85. 1

[8] Y. Y. Gambo F. Jarad, D. Baleanu, T. Abdeljawad, On Caputo modification of the Hadamard fractional derivatives, Adv. Difference Equ., 2014 (2014), 12 pages. 1, 3

[9] F. Gao, X.-J. Yang, Fractional Maxwell fluid with fractional derivative without singular kernel, Therm. Sci., 20 (2016), S871-S877. 1

[10] C. Goodrich, A. C. Peterson, Discrete fractional calculus, Springer, Cham, (2015). 1

[11] R. Hilfer (Ed.), Applications of fractional calculus in physics, World Scientific Publishing Co., Inc., River Edge, NJ, (2000). 1

[12] F. Jarad, T. Abdeljawad, D. Baleanu, Caputo-type modification of the Hadamard fractional derivatives, Adv. Difference Equ., 2012 (2012), 8 pages. 1, 3

[13] U. N. Katugampola, New approach to a generalized fractional integral, Appl. Math. Comput., 218 (2011), 860-865. 1, $2,3,3,3$

[14] U. N. Katugampola, A new approach to generalized fractional derivatives, Bull. Math. Anal. Appl., 6 (2014), 1-15. 1, 2.6

[15] A. A. Kilbas, Hadamard-type fractional calculus, J. Korean Math. Soc., 38 (2001), 1191-1204. 1, 2

[16] A. A. Kilbas, H. M. Srivastava, J. J. Trujillo, Theory and applications of fractional differential equations, North-Holland Mathematics Studies, Elsevier Science B.V., Amsterdam, (2006). 1, 2, 2, 3

[17] J. Losada, J. J. Nieto, Properties of a new fractional derivative without singular kernel, Progr. Fract. Differ. Appl., 1 (2015), 87-92. 1

[18] J. T. Machado, V. Kiryakova, F. Mainardi, Recent history of fractional calculus, Commun. Nonlinear Sci. Numer. Simul., 16 (2011), 1140-1153. 1

[19] R. L. Magin, Fractional calculus in bioengineering, Begell House Publishers, CT, (2006). 1

[20] I. Podlubny, Fractional differential equations, An introduction to fractional derivatives, fractional differential equations, to methods of their solution and some of their applications, Mathematics in Science and Engineering, Academic Press, Inc., San Diego, CA, (1999). 1, 3

[21] S. G. Samko, A. A. Kilbas, O. I. Marichev, Fractional integrals and derivatives, Theory and applications, Edited and with a foreword by S. M. Nikolskili, Translated from the 1987 Russian original, Revised by the authors, Gordon and Breach Science Publishers, Yverdon, (1993). 1

[22] X.-J. Yang, D. Baleanu, H. M. Srivastava, Local fractional integral transforms and their applications, Elsevier/Academic Press, Amsterdam, (2016). 1

[23] X.-J. Yang, F. Gao, J. A. Tenreiro Machado, D. Baleanu, A new fractional derivative involving the normalized sinc function without singular kernel, ArXiv, 2017 (2017), 11 pages. 1 